\title{
Community-based study on the knowledge, awareness, and practices of females towards breast cancer in Buraimi, Oman
}

\author{
Vijay Kumar Chattu, Soosanna Kumary', Akshaya Srikanth Bhagavathula ${ }^{2}$
}

\begin{abstract}
Background: In Oman, a higher proportion of patients presented with breast cancer are relatively young age and with an advanced stage. This could be due to lack of awareness and knowledge about breast cancer among young women. Aim: To assess the breast cancer knowledge, awareness and factors associated with the practice of breast self-examination (BSE) among females in Buraimi, Oman. Methods: A cross-sectional survey was conducted among females attending the two days health exhibition event at a polyclinc, in Al-Buraimi, governorate. A pretested questionnaire to assess their knowledge, awareness and practices concerned to breast cancer was used.The collected data was categorized, analyzed using descriptive statistics and logistic regression model.All the statistical analysis was performed using SPSS 21.0 version. Results: Out of the total of one hundred and eighty nine (I89) participants, $80 \%$ had information of breast cancer. Participants had better knowledge of symptoms but less knowledge about risk factors. When probed about BSE, only $43 \%$ performed it and only $21 \%$ of them do it monthly on regular basis. The main reasons for regular monthly BSE is mainly because of fear of breast cancer ( $9 \%$ ), doctor's advice (6\%) and awareness through media (5\%). Conclusion: Our study participants showed insufficient knowledge in some areas related to sign and symptoms and risk factors of breast cancer and identified a negative influence of low knowledge on the practice of BSE. Thus, educational interventions need to be emphasized to provide comprehensive information of breast cancer.
\end{abstract}

Key words: Awareness, breast cancer, breast self-examination, community-based, knowledge, Middle East, Oman, screening, young females

\section{Introduction}

Breast cancer is by far the most frequent cancer of women $(23 \%$ of all cancers), ranking second overall when both sexes are considered together. It is the leading cause of cancer mortality in women and constitutes $14 \%$ of female cancer deaths. ${ }^{[1]}$ The pattern of breast cancer in Arab countries is very disturbing. ${ }^{[2,3]}$ In Oman, breast cancer is the most frequent malignant tumor diagnosed in females, parallel to established global data, and on an average, $100-150$ new cases are reported annually. ${ }^{[4]}$ The primary factors that increase the risk of breast cancer in women include certain inherited genetic mutations, a personal or family history of breast cancer, and biopsy-confirmed hyperplasia. ${ }^{[5]}$ Since breast cancer is a progressive disease, small tumors are more likely to be at an early stage, and their early detection is more likely to have more successful treatment and a better prognosis. ${ }^{[6]}$ The three screening tests usually considered for early detection are clinical breast examination (CBE), X-ray mammography, and breast self-examination (BSE). ${ }^{[7,8]}$ In industrial countries, breast cancer mortality is declining where screening mammography is the standard for care. ${ }^{[9]} \mathrm{BSE}$ is appealing as a patient-centered, noninvasive screening procedure that allows women to become comfortable with their own bodies. ${ }^{[10]}$ Regular performance of BSE does not mean that the breast cancer is necessarily self-detected. BSE increases body awareness so that there is heightened awareness of changes that may be detected during BSE or at some other time. The aim of this study was to evaluate the knowledge, attitude, and practice of breast cancer screening behaviors among females in Buraimi, Oman.

\section{Materials and Methods}

\section{Study design and setting}

This is a cross-sectional study conducted on female participants who have consented to participate in this research irrespective of their age, marital status, and education was included in

\begin{tabular}{|c|}
\hline Access this article online \\
\hline 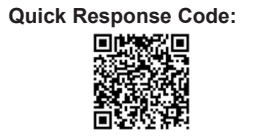 \\
\hline Website: www.sajc.org \\
\hline DOI: 10.4103/sajc.sajc_71_18 \\
\hline
\end{tabular}

Department of Paraclinical Sciences, Faculty of Medical Sciences, The University of the West Indies, St. Augustine, Trinidad and Tobago, 'Department of Paraclinical Sciences, Faculty of Medical Sciences, The University of the West Indies, St. Augustine, Trinidad and Tobago, ${ }^{2}$ Department of Clinical Pharmacy, University of Gondar-College of Medicine and Health Sciences, Gondar, Ethiopia

Correspondence to: Dr.Vijay Kumar Chattu, E-mail:vijay.chattu@sta.uwi.edu,drvkumar.ch@gmail.com the study. All the female participants who attended the health exhibition at Buraimi polyclinic were approached by the trained female interviewers from College of Health Sciences, Buraimi, Oman. All the female visitors, university students, and health workers attending this event were invited to participate in the survey. We included a total of 189 female participants who shared their responses during the study period were considered as our sample.

\section{Study questionnaire}

A predesigned self-administered questionnaire was used to collect the information from the teachers. The questionnaire was derived from other published studies dealing with the same topic as well as from our own experience. It included questions related to personal data and history of related health events. It also investigated the knowledge and awareness of teachers regarding breast cancer and their practice of screening procedures. The data collection process was made by the investigators themselves. The structured English form was first translated into Arabic by an author. This version was revised and translated back to English by another author and compared with the original form to ascertain the precision of translation. Knowledge about the risk of breast cancer was assessed by questionnaire items. These items included having had breast cancer once before, late age at first pregnancy, early onset of menstruation, having had a breast biopsy, having a family history of breast cancer, advanced age, and late-onset of menopause in addition to five items recently identified, namely breastfeeding, exposure to radiation, hormonal treatment, and high-fat diet and obesity.

The knowledge of women about the common screening methods (mammography, CBE, and BSE) was assessed by asking three questions. The source of knowledge was detected by asking the participant to define a source of her knowledge about breast cancer; it was permissible to give more than one source.

This is an open access journal, and articles are distributed under the terms of the Creative Commons Attribution-NonCommercial-ShareAlike 4.0 License, which allows others to remix, tweak, and build upon the work non-commercially, as long as appropriate credit is given and the new creations are licensed under the identical terms.

For reprints contact: reprints@medknow.com

How to cite this article: Chattu VK, Kumary S, Bhagavathula AS. Community-based study on the knowledge, awareness, and practices of females towards breast cancer in Buraimi, Oman. South Asian J Cancer 2018;7:215-8. 
A pilot study was carried out on 30 female students (not included in the final study). This study was formulated with the following objectives: test the clarity of the questions, test the validity and applicability of the study tools, accommodate the aim of the work to actual feasibility, identify the difficulties that may be faced during the application, as well as study all the procedures and activities of the administrative aspects.

\section{Ethical considerations}

Ethical approval was taken from the Institutional Ethical Board of College of Health Sciences, University of Buraimi. A written format explaining the purpose of the research was explained by the interviewer to the participant, and oral consent was taken before enrolling in the study. To maintain confidentiality, questionnaires were made anonymous.

\section{Statistical analysis}

The statistical package for the social sciences (SPSS Statistics for Windows, Version 19.0. Armonk, IBM Corp., NY) was used for data processing. Simple descriptive statistics were used (mean \pm standard deviation for quantitative variables, and frequency with percentage distribution for categorized variables). To find the most important factors considered as predictors of having satisfactory knowledge, a logistic regression analysis was used. A model was developed using all factors suspected to be associated with satisfactory knowledge level.

\section{Results}

A total of $189(n=189)$ participants agreed to participate in the study and shared their responses to the questions in the questionnaires. Table 1 shows general characteristics of the study population. A majority of the participants in this study were married $(n=119,63 \%)$ and the remaining were single $(n=68$, $36 \%)$ and two were widowed/divorced. Their age ranged between 21 and 58 years. Most participants $(87 \%)$ were aged $<40$ years and were ever married (64\%). Of the total 121 who were ever married, around two-thirds of the participants (79/121) were married for more than 5 years. Most of husbands had secondary or university education (82\%). About two-thirds $(67.5 \%)$ of the participants had information about breast cancer.

Participants' knowledge regarding the symptoms and signs of breast cancer is shown in Table 2. A higher proportion of participants responded correctly the presence of breast mass $(84 \%)$, breast pain $(77 \%)$, and abnormal enlargement of breast $(73.5 \%)$. Further, participants correctly identified smoking (74\%), consumption of fatty foods $(72.5 \%)$, family history of cancer $(72 \%)$, personal hygiene $(70 \%)$, and menopause age above 50 years $(67.7 \%)$ are some of the important risk factors.

Table 3 illustrates participants' knowledge and the frequency of application of breast examination. About three-fourth (73.5\%) declared that they do know how to practice BSE, 20.6\% expressed that they do self-examination of breast every month. In addition, $66 \%$ of the participants also underwent CBE, and only $14 \%$ reported that they underwent breast examination through mammography.

When requested the participants opinion about reasons of starting to perform BSE regularly, about $65.6 \%$ does not stated any reason, however, some participants stated its because of fear $(9 \%)$, doctors advise $(6.3 \%)$, and media broadcasting (5.3\%) [Table 4]. Further, when asked about reasons
Table 1: Demographic characteristics of the study participants $(n=189)$

\begin{tabular}{lc}
\hline Variables & $n(\%)$ \\
\hline Age groups & \\
$<30$ & $106(56)$ \\
$30-39$ & $59(31.2)$ \\
$\geq 40$ & $24(12.8)$ \\
Marital status & \\
Single & $68(36)$ \\
Divorced or widowed & $2(1)$ \\
Married & $119(63)$ \\
Duration of marriage & \\
$1-5$ & $42(22)$ \\
$6-10$ & $32(17)$ \\
$11-15$ & $20(10.6)$ \\
$>20$ & $9(5)$ \\
Husband's education & \\
Less than secondary & $21(11)$ \\
Secondary & $46(24.3)$ \\
University & $49(26)$ \\
Information about breast cancer & \\
Yes & $152(80)$ \\
No & $37(20)$ \\
Family history of breast cancer & \\
Yes & $156(82)$ \\
No & $33(18)$ \\
Total & $189(100)$ \\
\hline
\end{tabular}

Table 2: Participants knowledge about breast cancer $(n=189)$

\begin{tabular}{lc}
\hline Items & $n(\%)$ \\
\hline Symptoms and signs of breast cancer & \\
Bloody discharge from nipple & $138(73)$ \\
Presence of breast mass & $158(84)$ \\
Breast pain & $145(77)$ \\
Abnormal arm swelling & $125(66)$ \\
Nipple retraction & $122(65)$ \\
Abnormal enlargement of breast & $139(73.5)$ \\
Risk factors of breast cancer & \\
Aging & $123(65)$ \\
Nulliparity & $105(55.6)$ \\
Age of first delivery above 30 & $120(63.5)$ \\
Pregnancy at early age & $54(28.6)$ \\
Menopause age above 50 & $128(67.7)$ \\
Age of menarche under 11 years & $65(34)$ \\
Family history of cancer & $136(72)$ \\
Obesity & $117(62)$ \\
Breast feeding & $75(40)$ \\
Smoking & $140(74)$ \\
Sunlight exposure & $85(45)$ \\
Consumption of fatty foods & $137(72.5)$ \\
Consumption of spicy foods & $85(45)$ \\
Personal hygiene & $132(70)$ \\
\hline
\end{tabular}

for nonpracticing BSE regularly, participants' answers included lack of knowledge about BSE and its value (27\%), no breast complaints $(8.5 \%)$, fear of finding a lump $(4.8 \%)$, and no time for BSE $(2.1 \%)$ are some of the barriers reported by the them.

\section{Discussion}

This study demonstrated the knowledge and awareness of breast cancer among female participants ranged from little to adequate knowledge. The knowledge about the risk factors South Asian Journal of Cancer Volume 7 Issue 4 October-December 2018 
Table 3: Knowledge and awareness about breast examination

\begin{tabular}{lc}
\hline Method of examination & $n(\%)$ \\
\hline BSE & $139(73.5)$ \\
Yes & $51(27)$ \\
No & $50(26.5)$ \\
I do not know & $22(11.6)$ \\
Yes I apply whenever it comes my mind & $3(1.6)$ \\
Yes I apply once a week & $39(20.6)$ \\
Yes I apply once a month & $9(4.8)$ \\
Yes I apply every 2-4 month & $5(2.6)$ \\
Yes I apply every 5-6 month & $3(1.6)$ \\
Yes I apply once a year & \\
Clinical breast examination & $124(66)$ \\
Yes & $65(34)$ \\
No & \\
If yes, how often & $23(12)$ \\
Yearly & $12(6)$ \\
Every 2 years & $30(16)$ \\
Others & \\
Mammography & $26(14)$ \\
Yes & $163(86)$ \\
No & \\
If yes, how often & $9(4.8)$ \\
yearly & $4(2.1)$ \\
Every 2 years & $13(7)$ \\
Others &
\end{tabular}

BSE $=$ Breast self-examination

Table 4: Participants' opinions about reasons of starting and barriers for practicing breast self-examination regularly

\begin{tabular}{lc}
\hline Participant's opinion & $n(\%)$ \\
\hline Reasons of starting to perform BSE regularly & \\
Fear of breast cancer & $17(9)$ \\
Media & $10(5.3)$ \\
Doctor's advice & $12(6.3)$ \\
Breast pain & $6(3.2)$ \\
Advice of a health worker & $8(4.2)$ \\
Nipple discharge & $2(1.1)$ \\
The feeling of a mass & $2(1.1)$ \\
Breast cancer in the family & $4(2.1)$ \\
Encouraged by a friend & $4(2.1)$ \\
NA & $124(65.6)$ \\
Barriers for practicing BSE regularly & \\
Lack of knowledge (about BSE and its value) & $51(27)$ \\
Fear/worry to find a lump & $9(4.8)$ \\
No time for BSE & $4(2.1)$ \\
Forgetfulness & $3(1.6)$ \\
No breast complaints & $16(8.5)$ \\
Culture and health beliefs & $5(2.6)$ \\
Absence of lump during previous examination & $2(1.1)$ \\
Under estimate the problem of breast cancer & $4(2.1)$ \\
Others & $1(0.5)$ \\
NA & $94(49.7)$ \\
\hline NA-Not applicable BSE-Breast self-examination
\end{tabular}

NA=Not applicable, BSE=Breast self-examination

such as early age pregnancy, age of menarche under 11 years, nonbreastfeeding, and high sunlight exposure was not well recognized by the study participants. This might reflects the religious culture that encourages misconceptions concerning women opinions about the reasons of poor practicing of BSE. One of the major factors why most of the patients experience South Asian Journal of Cancer Volume 7 Issue 4 October-December 2018 late presentation of breast cancer because most of the women do not carry BSE and not aware of undergoing screening for BSE. In this study, $27 \%$ of the respondents have never heard about $\mathrm{BSE}$, while $66 \%$ of the respondents underwent $\mathrm{CBE}$ and only $14 \%$ were aware of mammography. This may be due to poor health education about the breast cancer in the society. Therefore, providing BSE education to all the females is warranted. Further, our respondents also have poor knowledge of the use of mammography as a screening tool for early detection of breast cancer. Health education about the benefits of mammography screening should be encouraged.

This study participants reported some of the barriers for participating BSE includes lack of knowledge and understanding of its value, thinking no breast complaints, and fear to find a lump. Our survey findings indicate that the women failed to obtain a mammography was the poor belief that examination is not important because of lack of educational programs to enhance women's perceived self-efficacy to have a mammography test.

\section{Conclusion}

The study points to the insufficient knowledge of the participants in some areas of knowledge related to sign and symptoms and barriers of breast cancer and identified the negative influence of low knowledge on the practice of BSE. Accordingly, relevant educational programs with the aim of improving the knowledge level of women regarding the breast cancer are needed considering the high prevalence of breast cancer among Omani females. At the same time, there is an urgent need for regular update courses for health staff, especially nurses about breast cancer education and various screening methods.

\section{Acknowledgment}

The authors would like to thank Dr. J.F Thomas, the Dean of College of Health Sciences and the University administration for their support and encouragement. The authors also thank all the female students of University of Buraimi and the women who made this study successful through their active participation and contribution

\section{Financial support and sponsorship}

Nil.

\section{Conflicts of interest}

There are no conflicts of interest.

\section{References}

1. Parkin DM, Bray F, Ferlay J, Pisani P. Global cancer statistics, 2002. CA Cancer J Clin 2005;55:74-108.

2. Amr SS, Sa'di AR, llahi F, Sheikh SS. The spectrum of breast diseases in Saudi Arab females: A 26 year pathological survey at Dhahran health center. Ann Saudi Med 1995; 15:125-32.

3. Mansoor I. Profile of female breast lesions in Saudi Arabia. J Pak Med Assoc 2001;51:243-7.

4. Chiedozi LC, El-Hag IA, Kollur SM. Breast diseases in the Northern region of Saudi Arabia. Saudi Med J 2003;24:623-7.

5. Mehdi I, Monem EA, Al Bahrani BJ, Al Kharusi S, Nada AM, Al Lawati J, et al. Age at diagnosis of female breast cancer in Oman: Issues and implications. South Asian J Cancer 2014;3:101-6.

6. Tabár L, Duffy SW, Vitak B, Chen HH, Prevost TC. The natural history of breast carcinoma: What have we learned from screening? Cancer 1999;86:449-62.

7. Sherma CD, Hossfeld DK. Breast cancer in manual of oncology. In: Bosch FX, editor. International Union Against Cancer: Middle East. $5^{\text {th }}$ ed. Berlin, Heidelberg, USA: Springer Verlag; 1990. p. 257-76.

8. Siahpush M, Singh GK. Sociodemographic predictors of pap test receipt, currency and knowledge among Australian women. Prev Med 
2002;35:362-8

9. Reynolds T. Declining breast cancer mortality: What's behind it? J Natl Cancer Inst 1999;91:750-3.
10. Vainio H, Bianchini F. Breast Cancer Screening: International Agency for Research on Cancer (IARC) Handbooks of Cancer Prevention. Vol. 7. Lyon (France): IARC Press; 2002. 